\title{
REGULAR SIMPLICES PASSING THROUGH HOLES
}

\author{
HIROSHI MAEHARA AND NORIHIDE TOKUSHIGE
}

\begin{abstract}
What is the smallest circular or square wall hole that a regular tetrahedron can pass? This problem was solved by Itoh-TanoueZamfirescu [8]. Then, we settled the case of equilateral triangular hole in [1]. Motivated by these results, we consider the corresponding problems in higher dimensions. Among other results, we determine the minimum $(n-1)$-dimensional ball hole that a unit regular $n$-simplex can pass. The diameter of the minimum hole goes to $3 \sqrt{2} / 4$ as $n$ tends to infinity.
\end{abstract}

\section{INTRODUCTION}

For a given convex body, find a small hole in a wall through which the convex body can pass. This type of problems goes back to Zindler [17] in 1920, who considered a convex polytope which can pass through a fairly small circular holes. A related topic known as Prince Rupert's problem can be found in [4]. Here we concentrate on the case when the convex body is a regular $n$-simplex.

For a compact convex body $K \subset \mathbb{R}^{n}$, let $\operatorname{diam}(K)$ and width $(K)$ denote the diameter and width of $K$, respectively. For $d>0$ let $d K$ denote the convex body of diameter $d \times \operatorname{diam}(K)$ and homothetic to $K$. By $S_{n}, Q_{n}$, and $B_{n}$ we denote the $n$-dimensional simplex, hypercube, and ball of unit diameter, respectively. Thus, $S_{n}$ has side length $1, Q_{n}$ has side length $1 / \sqrt{n}$, and $B_{n}$ has radius $1 / 2$.

Let $\Theta$ be an $(n-1)$-dimensional convex body with $\operatorname{diam}(\Theta)=1$ lying on a hyperplane in $\mathbb{R}^{n}$, which we will call a hole-shape. For a $d>0$, let $H$ be the hyperplane containing $d \Theta$. The hyperplane $H$ divides $\mathbb{R}^{n}$ into two (open) half spaces $H^{+}$and $H^{-}$. We want to push $S_{n}$ from $H^{+}$to $H^{-}$ through $d \Theta$. In this situation, we are interested in the following two types of minimum diameters $\gamma$ and $\Gamma$ of hole-shape $\Theta$, defined by

$$
\begin{aligned}
\gamma(n, \Theta) & :=\min \left\{d: S_{n} \text { can pass through the hole } d \Theta\right\}, \\
\Gamma(n, \Theta) & :=\min \left\{d: S_{n} \subset(d \Theta) \times \mathbb{R}\right\} .
\end{aligned}
$$

Date: April 5, 2010, 02:58pm.

2000 Mathematics Subject Classification. 52A20 and 52B11 and 52A40.

Key words and phrases. regular simplex, convex hole, diameter.

The authors were supported by MEXT Grant-in-Aid for Scientific Research (B) 20340022. 
Since $S_{n}$ can pass through a hole $d \Theta$ by translation perpendicular to the hyperplane containing the hole iff $d \geq \Gamma(n, \Theta)$, we have $\gamma(n, \Theta) \leq \Gamma(n, \Theta)$.

As we set the diameters of $Q_{n}, B_{n}$ equal to 1 , we have width $\left(Q_{n}\right)=1 / \sqrt{n}$ and width $\left(B_{n}\right)=1$. Steinhagen [14] determined the width of $S_{n}$ as follows.

$$
\text { width }\left(S_{n}\right)= \begin{cases}\sqrt{\frac{2}{n+1}} & \text { if } n \text { is odd } \\ \sqrt{\frac{2 n+2}{n(n+2)}} & \text { if } n \text { is even. }\end{cases}
$$

If $S_{n}$ can pass through a hole $d \Theta$ by translation, then

$$
\operatorname{width}(d \Theta) \geq \operatorname{width}\left(S_{n}\right)=(\sqrt{2}-o(1)) / \sqrt{n} .
$$

Let $n \geq 3$. If $S_{n}$ can pass through a hole $d \Theta$, then $d \geq \operatorname{width}\left(S_{2}\right)=\sqrt{3} / 2$. This gives $\gamma(n, \Theta) \geq \sqrt{3} / 2$.

The following result is due to Pukhov [12] and Weißback [15] for $n$ odd, and Brandenberg and Theobald [2, 3] for $n$ even (and odd).

$$
\Gamma\left(n, B_{n-1}\right)= \begin{cases}\sqrt{\frac{2(n-1)}{n+1}} & \text { if } n \text { is odd } \\ \frac{2 n-1}{\sqrt{2 n(n+1)}} & \text { if } n \text { is even. }\end{cases}
$$

In the next section, we review some results in $\mathbb{R}^{3}$. Then, in section 3 we consider the problems in higher dimensions. Among other results, we will show that

and

$$
\begin{gathered}
\lim _{n \rightarrow \infty} \gamma\left(n, S_{n-1}\right)=\lim _{n \rightarrow \infty} \Gamma\left(n, S_{n-1}\right)=1, \\
\lim _{n \rightarrow \infty} \gamma\left(n, B_{n-1}\right)=3 \sqrt{2} / 4, \quad \lim _{n \rightarrow \infty} \Gamma\left(n, B_{n-1}\right)=\sqrt{2},
\end{gathered}
$$

$$
3 \sqrt{2} / 4 \leq \lim _{n \rightarrow \infty} \gamma\left(n, Q_{n-1}\right) \leq 2, \quad \sqrt{2} \leq \lim _{n \rightarrow \infty} \Gamma\left(n, Q_{n-1}\right) \leq 2 .
$$

It would be also interesting to consider problems concerning a general $n$ simplex of diameter 1 passing through holes.

\section{IN THE 3-SPACE}

Itoh, Tanoue, and Zamfirescu [8] proved

$$
\gamma\left(3, Q_{2}\right)=\Gamma\left(3, Q_{2}\right)=1, \quad \gamma\left(3, B_{2}\right)=2 r=0.8956 \ldots,
$$

where $r \in(0,1)$ is a unique root of the equation $216 x^{6}-9 x^{4}+38 x^{2}-9=0$. We note that $\gamma\left(3, B_{2}\right)<\Gamma\left(3, B_{2}\right)=1$.

In [1], the following is proved.

$$
\gamma\left(3, S_{2}\right)=\Gamma\left(3, S_{2}\right)=\frac{1+\sqrt{2}}{\sqrt{6}}=0.9855 \ldots
$$

Zamfirescu [16] proved that most convex bodies can be held by a circular frame. Using (4), one can show that a square frame of diagonal length $d$ 
can hold $S_{3}$ iff $1 / \sqrt{2}<d<1$, and a circular frame of diameter $d$ can hold $S_{3}$ iff $1 / \sqrt{2}<d<\gamma\left(3, B_{2}\right)$, see [8].

On the other hand, it is shown in [1] that

$$
\text { no triangular frame can hold a convex body. }
$$

This is a special property for triangular frames, and in fact, every nontriangular frame holds some tetrahedron in $\mathbb{R}^{3}$, see [1].

According to Debrunner and Mani-Levitska [5], Janos Pach asked the following: "If a convec body (say, a stone) can be thrust through a convex hole in a wall by a linear motion (without twisting) then can this be done by a movement perpendicular to the wall?" The answer is positive, and it is proved in [5] that any section of a right cylinder by a plane contains a congruent copy of the base, see also [10], [9]. This together with (5) implies the following: if a convex body, not necessarily smooth, can pass through a triangular hole, then the convex body can pass through the hole by translation perpendicular to the wall, see [1].

Itoh and Zamfirescu [7] found a hole $\Phi$ on the plane in $\mathbb{R}^{3}$ with $\operatorname{diam}(\Phi)=$ $\operatorname{width}\left(S_{2}\right)=\sqrt{3} / 2$ and width $(\Phi)=\operatorname{width}\left(S_{3}\right)=\sqrt{2} / 2$, such that $S_{3}$ can pass through $\Phi$.

\section{HIGHER DIMENSIONS}

3.1. Holes of shape $S_{n-1}$. It is known from [5, 9] that any plane section of a right triangular prism contains a congruent copy of a base of the prism. The situation in higher dimension is different. In [5], it is proved that if $n>3$, then for any right cylinder with convex polytope base, one can find a hyperplane section which does not contain a congruent copy of the base. Nevertheless, we have the following.

Theorem 1. Let $K \subset \mathbb{R}^{n}$ be a compact convex body, and let $\Delta_{n-1}$ be a general $(n-1)$-simplex. If $K$ can pass through the hole $\Delta_{n-1}$, then this can be done by translation only.

Proof. First consider the case that $K$ is a smooth convex body, that is, the case that, for every point $x$ on the boundary $\partial K$ of $K$, there is a unique supporting hyperplane of $K$ tangent to $K$ at $x$. Let $\Delta_{n-1}=p_{1} \ldots p_{n}$ be the hole on the hyperplane $H$, and $H_{+}, H_{-}$be the half spaces such that $H_{+} \cap$ $H_{-}=H$. Let $f: K \times[0,1] \rightarrow \mathbb{R}^{n}$ be a continuous motion of $K$ such that $f(K, 0) \subset H_{-}, f(K, 1) \subset H_{+}$, and $H \cap f(K, t) \subset \Delta_{n-1}$ for all $0<t<1$. We may further suppose that, for $0<t<1, f(K, t)$ touchs all facets of $\Delta_{n-1}$ except the facet opposite to the vertex $p_{n}$. Let $f(K, t)_{-}=f(K, t) \cap H_{-}$and $f(K, t)_{+}=f(K, t) \cap H_{+}$.

Now, for $0<t<1$ and $1 \leq i<n$, let $H(i, t)$ denote the supporting hyperplane of $f(K, t)$ containing the facet of $\Delta_{n-1}$ opposite to $p_{i}$. Then the 
intersection $L(t)=H(1, t) \cap H(2, t) \cap \cdots \cap H(n-1,1)$ is a line. Let $H(n, t)$ be the hyperplane parallel to the line $L(t)$ and containig the facet of $\Delta_{n-1}$ opposite to $p_{n}$. Then the $n$ hyperplanes $H(1, t), \ldots, H(n, t)$ determine a prism $P(t)$ that intersects $H$ at $\Delta_{n-1}$. Note that, for each $1 \leq i<n$ and $0<t<1$, $f(K, t)$ and $P(t)$ lie in the same side of $H(i, t)$. Furthermore, since $f(K, t)$ is convex, one of $f(K, t)_{-}, f(K, t)_{+}$lies in the same side of $H(n, t)$ as $P$. Therefore, one of $f(K, t)_{-}, f(K, t)_{+}$is contained in $P(t)$. We may suppose that $f(K, t)_{+} \subset P(t)$ for $t<\delta$, and $f(K, t)_{-} \subset P(t)$ for $t>1-\delta$, where $\delta$ is some fixed small positive constant.

Let $t_{0}=\max \left\{t: f(K, t)_{+} \subset P(t), \delta \leq t \leq 1-\delta\right\}$. Then, for every $\varepsilon>0$, $f\left(K, t_{0}+\varepsilon\right)_{-} \subset P\left(t_{0}+\varepsilon\right)$. Since $f(K, t)$ and each hyperplane $H(i, t)(1 \leq$ $i \leq n)$ move continuously on $t$, this implies that $f\left(K, t_{0}\right)_{-} \subset P\left(t_{0}\right)$, and hence $f\left(K, t_{0}\right) \subset P\left(t_{0}\right)$. Thus the prism $P\left(t_{0}\right)$ contains a congruent copy of $K$, and hence $K$ can pass through $\Delta_{n-1}$ by a translation.

Next, let us consider the case that the convex body $K$ is not smooth. We may suppose that $K$ contains the origin 0 of $\mathbb{R}^{n}$. For $r>0$, let $B(r)$ denote the ball with center 0 and radius $r$. Then, for each integer $k>0$, $K+B(1 / k)$ is a smooth convex body, and if $K$ can pass through $\Delta_{n-1}$, then $K+B(1 / k)$ can pass through the hole of $(1+2 / k) \Delta_{n-1}$. Hence there is a prism $P_{k}$ containing $K+B(1 / k)$ and $P_{k} \cap H=(1+2 / k) \Delta_{n+1}$, where $H$ is the hyperplane containing $\Delta$. Let $r_{0} \gg \operatorname{diam}(K)$, and put $X_{k}=P_{k} \cap B\left(r_{0}\right)$. Then, in $\left\{X_{k}: k=1,2, \ldots\right\}$, Blaschke selection theorem guarantees the existence of a subsequence $\left\{X_{k_{m}}\right\}$ and a convex set $X_{\infty}$ such that $X_{k_{m}}$ converges to $X_{\infty}$ in the Hausdorff metric. This $X_{\infty}$ determines a prism $P_{\infty}$ with $P_{\infty} \cap H=\Delta_{n-1}$ such that $K \subset P_{\infty}$. Hence $K$ can pass through $\Delta_{n-1}$ by a translation.

Problem 1. Is it possible to take the translation in Theorem Пperpendicular to the wall? Or equivalently, do $\gamma\left(n, S_{n-1}\right)$ and $\Gamma\left(n, S_{n-1}\right)$ coincide?

Theorem 2.

$$
\gamma\left(n, S_{n-1}\right) \geq \begin{cases}\sqrt{1-\frac{1}{n}} & \text { if } n \text { is odd } \\ \sqrt{1-\frac{1}{n+2}} & \text { if } n \text { is even. }\end{cases}
$$

Proof. Suppose that $S_{n}$ can pass through the hole of $d S_{n-1}$. By Theorem 1 , this can be done by translation only. Thus we can apply (2) with (1), which implies the desired inequality.

The above result together with $\gamma\left(n, S_{n-1}\right) \leq \Gamma\left(n, S_{n-1}\right) \leq 1$ gives

$$
\lim _{n \rightarrow \infty} \gamma\left(n, S_{n-1}\right)=\lim _{n \rightarrow \infty} \Gamma\left(n, S_{n-1}\right)=1 .
$$

If the simplex does pass through a hole, then in particular the volume of some central hyperplane section of that simplex is no bigger than the volume of the hole. Jiří Matoušek suggested showing $\gamma\left(n, S_{n-1}\right) \rightarrow 1$ by using 
this simple observation. Then, Matthieu Fradelizi told us that a result in [6] implies that the volume of the smallest central hyperplane section of $S_{n}$ is more than $\operatorname{vol}\left(S_{n-1}\right) /(2 \sqrt{3})$, and this is enough for proving $\gamma\left(n, S_{n-1}\right) \rightarrow 1$. It is conjectured that the smallest central hyperplane section of $S_{n}$ is obtained by a hyperplane parallel to a facet of the simplex.

Since the diameter of circumsphere of $S_{n}$ is $\sqrt{2(n-1) / n}$, we have

$$
\Gamma\left(n, S_{n-1}\right) \sqrt{\frac{2(n-1)}{n}} \geq \Gamma\left(n, B_{n-1}\right) .
$$

This together with (3) implies

$$
\Gamma\left(n, S_{n-1}\right) \geq \sqrt{1-\frac{1}{n+1}}
$$

for $n$ odd. (For $n$ even, Theorem 2 gives a better lower bound for $\Gamma\left(n, S_{n-1}\right)$.)

Actually $S_{n}$ can pass through a hole smaller than its facet.

Theorem 3. $\Gamma\left(n, S_{n-1}\right)<1$ for all $n \geq 2$.

Before the formal proof, let us try the case $n=3$ to get a feel. Let $S_{2}=$ $A_{0} A_{1} A_{2}, A_{0}=(0,1 / 2), A_{1}=(0,-1 / 2), A_{2}=(\sqrt{3} / 2,0)$, and let $\mathscr{P}$ be the right triangular prism with base $A_{0} A_{1} A_{2}$. We put the unit regular tetrahedron $S_{3}=B_{0} B_{1} B_{2} B_{3}$ in the prism, namely, we set

$$
B_{0}=(0,1 / 2,0), B_{1}=(0,-1 / 2,0), B_{2}=(1 / \sqrt{2}, 0,1 / 2), B_{3}=(1 / \sqrt{2}, 0,-1 / 2) .
$$

Now we move the tetrahedron very slightly keeping it inside $\mathscr{P}$ so that all vertices are off the faces of $\mathscr{P}$. This can be done by rotating the tetrahedron along the $x$-axis, and push it in the direction of $x$-axis. This gives $\Gamma\left(3, S_{2}\right)<$ 1.

Proof. For $n \geq 2$, let

$$
S_{n}=A_{0} A_{1} \cdots A_{n} \subset \mathbb{R}^{n}
$$

be a unit regular $n$-simplex with vertices $A_{0}, \ldots, A_{n}$. We may assume that

$$
\begin{aligned}
& A_{i}=(0, *, \ldots, *) \in \mathbb{R}^{n} \text { for } 0 \leq i<n, \\
& A_{n}=(h, 0, \ldots, 0) \in \mathbb{R}^{n},
\end{aligned}
$$

where $h=\sqrt{(n+1) /(2 n)}$ is the height of $S_{n}$. Let $S_{n-1}$ be the facet spanned by $A_{0}, \ldots, A_{n-1}$. We may assume that the center of $S_{n-1}$ coincides with the origin.

Then we can construct a unit regular $(n+1)$-simplex

$$
S_{n+1}=B_{0} B_{1} \cdots B_{n} B_{n+1} \subset \mathbb{R}^{n+1}
$$


by setting

$$
\begin{aligned}
B_{i} & =\left(A_{i}, 0\right) \in \mathbb{R}^{n+1} \text { for } 0 \leq i<n, \\
B_{n} & =(k, 0, \ldots, 0,1 / 2) \in \mathbb{R}^{n+1}, \\
B_{n+1} & =(k, 0, \ldots, 0,-1 / 2) \in \mathbb{R}^{n+1},
\end{aligned}
$$

where $k=\sqrt{h^{2}-(1 / 4)}$. Since $0<k<h$ we notice that this $S_{n+1}$ is contained in the prism $\mathscr{P}=S_{n} \times \mathbb{R}$. More precisely, two vertices $B_{n}$ and $B_{n+1}$ are interior points of $\mathscr{P}$, while a face $F=B_{0} B_{1} \cdots B_{n-1}$ is contained in a facet $S_{n-1} \times \mathbb{R}$ of $\mathscr{P}$.

We will show by induction on $n$ that we can continuously move our $S_{n+1}$ inside the prism $\mathscr{P}$ so that $S_{n+1}$ will be contained in $\mathscr{P} \circ$, where $\mathscr{P}^{\circ}$ denotes the interior of $\mathscr{P}$. Namely, we will find a continuous function $f_{n+1}:[0,1] \times \mathbb{R}^{n+1} \rightarrow \mathbb{R}^{n+1}$ and $t_{0} \in(0,1)$ with $f_{n+1}\left(0, S_{n+1}\right)=S_{n+1}$ (the starting position) and $f_{n+1}\left(t, S_{n+1}\right) \subset \mathscr{P}^{\circ}$ for all $t \in\left(0, t_{0}\right]$.

For the base case, we take

$$
f_{3}(t, x, y, z)=(x+t, y \cos t-z \sin t, y \sin t+z \cos t),
$$

namely, we rotate the regular tetrahedron along the $x$-axis and push it in the direction of $x$-axis. Now let $n \geq 3$ and apply the induction hypothesis to the $(n-1)$-simplex $F$ with the prism $\mathscr{P}^{\prime}=F \times \mathbb{R}$. Then we can find a continuous function $f_{n}$ and $t_{0}^{\prime}$ with the required properties, in particular, $f_{n}(t, F) \subset\left(\mathscr{P}^{\prime}\right)^{\circ}$ for $t \in\left(0, t_{0}^{\prime}\right]$. We extend $f_{n}$ to define

$$
f_{n+1}\left(t, x_{0}, x_{1}, \ldots, x_{n}\right)=\left(x_{0}+t, f_{n}\left(t, x_{1}, \ldots, x_{n}\right)\right) .
$$

Then we have $f_{n+1}\left(0, S_{n+1}\right)=S_{n+1}, f_{n+1}\left(0,\left\{B_{n}, B_{n+1}\right\}\right) \subset \mathscr{P} \circ$ and

$$
f_{n+1}(t, F) \subset(t, 0, \ldots, 0)+\left(\mathscr{P}^{\prime}\right)^{\circ} \text { for } t \in\left(0, t_{0}^{\prime}\right] .
$$

Thus, we can choose $0<t_{0} \ll t_{0}^{\prime}$ such that $f_{n+1}\left(t, S_{n+1}\right)=f_{n+1}(t, F \cup$ $\left.\left\{B_{n}, B_{n+1}\right\}\right) \subset \mathscr{P}^{\circ}$ for $t \in\left(0, t_{0}\right]$.

3.2. Holes of shape $B_{n-1}$. We have $\Gamma\left(n, B_{n-1}\right) \rightarrow \sqrt{2}$ as $n \rightarrow \infty$ by (3). On the other hand, we will show $\gamma\left(n, B_{n-1}\right) \rightarrow 3 \sqrt{2} / 4$. Namely, rotation does help for escaping through a round hole.

By a hyperdisk we mean an $(n-1)$-ball sitting on a hyperplane in $\mathbb{R}^{n}$. We say that a hyperplane $H$ cuts a line segments $x y$ if $H$ separates $x$ and $y$. A hyperdisk $D \subset H$ is said to cut a line segment $x y$ if $H$ cuts $x y$ and $D$ intersects $x y$. In this case, the intersection of the hyperdisk $D$ and the line segment $x y$ is a singleton. The radius of a hyperdisk $D$ is denoted by $r(D)$.

Let $S_{n} \subset \mathbb{R}^{n}$ be a unit regualr $n$-simplex with vertex set $V\left(S_{n}\right)$. Choose $p \in V\left(S_{n}\right)$. By a proper partition, we mean to partition $V\left(S_{n}\right)$ into three nonempty sets $V\left(S_{n}\right)=\{p\} \cup X \cup Y$. Then, $X$ and $Y$ are divided by a hyperplane. Let $b_{X}, b_{Y}$ be the barycenters of $X, Y$, respectively. For $0<s<1$, 
let

$$
z_{s}=(1-s) b_{X}+s b_{Y}
$$

This point divides the line segment $b_{X} b_{Y}$ into $s: 1-s$. Let $H_{p}$ denote the hyperplane containing $V\left(S_{n}\right) \backslash\{p\}$, and let $I(X, Y, s) \subset H_{p}$ be the $(n-2)$ dimensional flat passing through $z_{s}$ and perpendicular to $b_{X} b_{Y}$. For each $-1 \leq t<1$, let

$$
w_{t}= \begin{cases}(1-t) p+t b_{Y} & \text { if } 0 \leq t<1 \\ (1-|t|) p+|t| b_{X} & \text { if }-1 \leq t<0 .\end{cases}
$$

Let $H(X, Y, s, t)$ denote the hyperplane obtained by rotating $H_{p}$ around $I(X, Y, s)$ so that it comes to the position where it intersects $p b_{X}$ or $p b_{Y}$ at the point $w_{t}$. Since $\operatorname{dim} I(X, Y, s)=n-2$, it is possible to rotate $H_{p}$ around $I(X, Y, s)$. We sometimes use a redundant notation $I_{p}(X, Y, s)$ or $H_{p}(X, Y, s, t)$ to emphasize that $p$ is the chosen vertex.

For $x \in X$ and $y \in Y$, we notice that $H(X, Y, s, t)$ divides the edge $x y$ with ratio $s: 1-s$, and it divides the edge $p x$ or $p y$ with ratio $t: 1-t$ (resp. $|t|: 1-|t|)$ if $t \geq 0$ (resp. if $t<0$ ). The radius of the smallest hyperdisk on $H(X, Y, s, t)$ that contains $S_{n} \cap H(X, Y, s, t)$ will be denoted by

$$
r(|X|, s, t),
$$

which only depends on $s, t$ and $|X|$, the cardinality of $X$.

Lemma 1. Let $V\left(S_{n}\right)=\{p\} \cup X \cup Y$ be a proper partition. Suppose that $D$ is a $(p, X, Y)$-hyperdisk, that is, $D$ contains $p$, and $D$ cuts all edges $x y$, where $x \in X, y \in Y$. Then $r(|X|, s, 0) \leq r(D)$ holds for some $0<s<1$.

Proof. For a $(p, X, Y)$-hyperdisk $D$, let $D_{x y}$ denote the point where $D$ cuts the edge connecting $x \in X$ and $y \in Y$. For $X^{\prime} \subset X, Y^{\prime} \subset Y$, let

$$
\begin{aligned}
& d\left(D, X^{\prime}, Y^{\prime}\right)=\left\{\left|x-D_{x y}\right|: x \in X^{\prime}, y \in Y^{\prime}\right\}, \\
& \delta\left(D, X^{\prime}, Y^{\prime}\right)=\max d\left(D, X^{\prime}, Y^{\prime}\right)-\min d\left(D, X^{\prime}, Y^{\prime}\right) .
\end{aligned}
$$

We define the irregularity of $D$ by $\delta(D):=\delta(D, X, Y)$. If $\delta(D)=0$, namely, if $d(D, X, Y)=\{s\}$ for some $0<s<1$, then each $D_{x y}$ lies on $H(X, Y, s, 0)$ and $r(|X|, s, 0) \leq r(D)$. So we may assume that $\delta(D)>0$. Let $\delta_{0}$ be the infimum of the irregularities of $(p, X, Y)$-hyperdisks with radius at most $r(D)$. By applying Blaschke selection theorem, we can find a $(p, X, Y)$-hyperdisk $D_{\infty}$ with $r\left(D_{\infty}\right) \leq r(D)$ and $\delta\left(D_{\infty}\right)=\delta_{0}$. Then, the following claim implies that $\delta_{0}=0$, which completes the proof of the lemma.

Claim 1. If $\delta(D)>0$, then there is a $(p, X, Y)$-hyperdisk $D^{*}$ with $r\left(D^{*}\right) \leq$ $r(D)$ and $\delta\left(D^{*}\right)<\delta(D)$. 
Now we prove the claim by introducing an operation which rotates $D$ to get $D^{*}$. Suppose that

$$
\delta(D)=\left|a-D_{a c}\right|-\left|b-D_{b d}\right|>0,
$$

where $a, b \in X$ and $c, d \in Y$. Let $K$ be the hyperplane contianing $D$. Then the line $a b$ or $c d$ intersects $K$. By symmetry, we may assume that the line $a b$ intersects $K$. Let $L$ be the hyperplane that perpendicularly bisects the edge $a b$, and let $J=K \cap L$. Since $\operatorname{dim} J=d-2$, we can rotate $K$ around $J$ until it comes to the position where the hyperplane becomes parallel to the line $a b$. Let $K^{*}$ be the resulting hyperplane. Let $D^{\prime}$ be the mirror image of $D$ with respect to $L$, and let $B$ be the minimum ball containing $D \cup D^{\prime}$. Finally let $D^{*}=B \cap K^{*}$. Since the distance from the center of $B$ to $J(\subset K)$ is greater than or equal to the distance between the center of $B$ and $K$, it follows that $r\left(D^{*}\right) \leq r(D)$.

We will show that $D^{*}$ is a $(p, X, Y)$-hyperdisk with $\delta\left(D^{*}\right)<\delta(D)$. Let $x \in X \backslash\{a, b\}$ and $y \in Y$. Since $V\left(S_{n}\right) \backslash\{a, b\}$ lies on $L$, we have $D_{x y} \in$ $D \cap L=D \cap K \cap L=D \cap J \subset D^{*}$, and $D_{x y}=D_{x y}^{*}$. This gives

$$
\delta(D, X \backslash\{a, b\}, Y)=\delta\left(D^{*}, X \backslash\{a, b\}, Y\right) .
$$

If $x \in\{a, b\}$ and $y \in Y$, then $K^{*}$ cuts the line segment $D_{x y} D_{x y}^{\prime}$, because both $D$ and $D^{\prime}$ are $(p, X, Y)$-hyperdisks. Thus the intersection is inside $B$ and we can write it $D_{x y}^{*}$. We may assume that the line $a b$ intersects $K$ at the extention beyond $b$. Since $D_{a y}^{*} D_{b y}^{*} \| a b$ and two edges $D_{a y}^{*} D_{b y}^{*}$ and $D_{a y} D_{b y}$ meet inside the equilateral $\triangle a b y$, we have $\left|a-D_{a y}\right|>\left|a-D_{a y}^{*}\right|$, and $\mid b-$ $D_{b y}|<| b-D_{b y}^{*} \mid$. Thus we have

$$
\delta(D,\{a, b\}, Y)>\delta\left(D^{*},\{a, b\}, Y\right) .
$$

If $\delta(D)=\delta(D, X \backslash\{a, b\}, Y)$, then we have succeeded to decrease the number of pairs $\{a c, b d\}$ which attain (6) by changing from $D$ to $D^{*}$. So, by repeating this operation, we may assume that $\{a c, b d\}$ is the only pair which attains (6), or equivalently, $\delta(D)>\delta(D, X \backslash\{a, b\}, Y)$. In this case, we have $\delta(D)>\delta\left(D^{*}\right)$ as desired.

For computation, it is convenient to put our $S_{n}$ in $\mathbb{R}^{n+1}$ instead of $\mathbb{R}^{n}$. So let $S_{n}=p_{1} p_{2} \cdots p_{n+1} \subset \mathbb{R}^{n+1}$ with $p_{j}=e_{j} / \sqrt{2}$, where $e_{j}$ is the $j$-th standard base of $\mathbb{R}^{n+1}$. Fix $s, t \in(0,1)$ and $1 \leq i<n$. Let $V\left(S_{n}\right)=\{p\} \cup$ $X \cup Y$ be a proper partition with $|X|=i$. To compute $r(i, s, t)$, we look at the hyperplane $H:=H_{p}(X, Y, s, t)$. Recall that $b_{X}, b_{Y}$ are the barycenters of $X$, $Y$, respectively. Fix two vertices $x \in X, y \in Y$. Let $u$ be the intersection of $x y$ and $H$, and let $\alpha=\left|u-z_{s}\right|$, where $z_{s}=(1-s) b_{X}+s b_{Y}$. Then we have

$$
2 \alpha^{2}=(1-s)^{2}\left(1-\frac{1}{i}\right)+s^{2}\left(1-\frac{1}{n-i}\right) \text {. }
$$


Let $v$ be the intersection of $p y$ and $H$, and let $\beta=\left|v-w_{t}\right|$, where $w_{t}=$ $(1-t) p+t b_{Y}$. Then we have

$$
2 \beta^{2}=t^{2}\left(1-\frac{1}{n-i}\right) .
$$

Notice that the distances $\alpha, \beta$ are independent of the choices of $x, y$. Also one can see that the two lines $u z_{s}$ and $v w_{t}$ are both perpendicular to $z_{s} w_{t}$. The distance $\lambda=\left|z_{s}-w_{t}\right|$ satisfies

$$
2 \lambda^{2}=\frac{(1-s)^{2}}{i}+(1-t)^{2}+\frac{(s-t)^{2}}{n-i} .
$$

Let $D$ be the smallest hyperdisk on $H$ that contains $S_{n} \cap H$, and let $o$ be its center. Then $o$ is on the line segment $z_{s} w_{t}$, and $|u-o|=|v-o|=r(i, s, t)$. Let $\zeta=\left|z_{s}-o\right|$. Then, looking at two right triangles $\triangle o u z_{s}$ and $\triangle o v w_{t}$, we have $r(i, s, t)^{2}=\alpha^{2}+\zeta^{2}=(\lambda-\zeta)^{2}+\beta^{2}$. This gives $\zeta=\left(\alpha^{2}-\beta^{2}-\right.$ $\left.\lambda^{2}\right) /(2 \lambda)$, and

$$
r(i, s, t)^{2}=\alpha^{2}+\frac{1}{4 \lambda^{2}}\left(\lambda^{2}-\alpha^{2}+\beta^{2}\right)^{2} .
$$

By substituting (7), (8), (9) into (10), we can rewrite $r(i, s, t)^{2}$ in terms of $s, t, i$ (and $n$ ) only. We record a special case for later use:

$$
2 r(i, s, 0)^{2}=\frac{i(n-i)\left(s^{2}-s+1\right)^{2}}{(1-s)^{2} n+(n+2 s-1) i-i^{2}} .
$$

Lemma 2. Let $V\left(S_{n}\right)=\{p\} \cup X \cup Y$ be a proper partition with $|X|=i$, and let $0<s<1$. Then we have $r(i, s, t) \leq r(i, s, 0)$ for all $t \in[s-1, s]$.

Proof. First assume that $0<t \leq s$. Let $D^{\prime}$ be the hyperdisk with center $o^{\prime}$ on $H(X, Y, s, 0)$ that is obtained by rotating $D$ around $I(X, Y, s)$ through the angle $\angle w_{t} z_{s} p$. Notice that $o^{\prime}$ is on the line segment $z_{s} p$, and $\left\{u, z_{s}\right\} \subset D \cap D^{\prime}$. Since $r(i, s, t)=r(D)=|u-o|$, to show $r(i, s, t)<r(i, s, 0)$ it suffices to show $p \notin D^{\prime}$, or equivalently, $\left|p-o^{\prime}\right|>r\left(D^{\prime}\right)=r(D)$.

Recall that $\alpha=\left|u-z_{s}\right|, \beta=\left|v-w_{t}\right|, \lambda=\left|z_{s}-w_{t}\right|, \zeta=\left|z_{s}-o\right|=\left|z_{s}-o^{\prime}\right|$, and $\left|w_{t}-o\right|=\lambda-\zeta$. Let $\eta=\left|p-w_{t}\right|$.

In $\triangle p b_{X} b_{Y}$, the edge $b_{X} b_{Y}$ is the shortest one. In fact, we have $\mid p-$ $\left.b_{X}\right|^{2}=\frac{1}{2}\left(1+\frac{1}{i}\right),\left|p-b_{Y}\right|^{2}=\frac{1}{2}\left(1+\frac{1}{n-i}\right)$, and $\left|b_{X}-b_{Y}\right|^{2}=\frac{1}{2}\left(\frac{1}{i}+\frac{1}{n-i}\right)$. Hence $\angle b_{X} p b_{Y}<\pi / 3$. Since $z_{s} w_{s} \| b_{X} p$, we have $\angle z_{s} w_{t} p \geq \angle z_{s} w_{s} p>$ $2 \pi / 3$. Looking at $\triangle z_{s} w_{t} p$, we get $\left|p-z_{s}\right|^{2}>\lambda^{2}+\eta^{2}+\lambda \eta$. Thus we have

$$
\left|p-o^{\prime}\right|=\left|p-z_{s}\right|-\left|z_{s}-o^{\prime}\right|>\sqrt{\lambda^{2}+\eta^{2}+\lambda \eta}-\zeta .
$$

Using $\triangle p v w_{t} \sim \triangle p y b_{Y}$, we have

$$
\left|v-w_{t}\right|=t\left|y-b_{Y}\right|=\frac{t}{2}\left(1-\frac{1}{n-i}\right)<\frac{t}{2}\left(1+\frac{1}{n-i}\right)=t\left|p-b_{Y}\right|=\left|p-w_{t}\right|=\eta,
$$


which implies

$$
r(D)^{2}=|v-o|^{2}=\left|v-w_{t}\right|^{2}+\left|w_{t}-o\right|^{2}<\eta^{2}+(\lambda-\zeta)^{2} .
$$

Since $0<t \leq s<1$, it follows from (7) and (8) that $\left|u-z_{s}\right|=\alpha>$ $\beta=\left|v-w_{t}\right|$. Then looking at two triangles $\triangle o u z_{s}$ and $\triangle o v w_{t}$ having hypotenuses of the same length $(=r(D))$, we get $\left|z_{s}-o\right|<\left|w_{t}-o\right|$, that is,

$$
2 \zeta<\lambda
$$

By (12) (13) and (14), we have

$$
\begin{aligned}
\left|p-o^{\prime}\right|^{2}-r(D)^{2} & >\left(\sqrt{\lambda^{2}+\eta^{2}+\lambda \eta}-\zeta\right)^{2}-\left(\eta^{2}+(\lambda-\zeta)^{2}\right) \\
& =\lambda \eta-2 \zeta\left(\sqrt{\lambda^{2}+\eta^{2}+\lambda \eta}-\lambda\right) \\
& >\lambda\left(\lambda+\eta-\sqrt{\lambda^{2}+\eta^{2}+\lambda \eta}\right)>0,
\end{aligned}
$$

as desired.

Next assume that $s-1 \leq t<0$. By comparing two proper partitions $\{p\} \cup$ $X \cup Y$ and $\{p\} \cup X^{\prime} \cup Y^{\prime}$, where $X^{\prime}=Y$ and $Y^{\prime}=X$, we have $r(|X|, s, t)=$ $r\left(\left|X^{\prime}\right|, 1-s,-t,\right)$. Then, using the inequality for $0<-t \leq 1-s<1$, we have $r(i, s, t)=r(n-i, 1-s,-t) \leq r(n-i, 1-s, 0)=r(i, s, 0)$, as desired.

Lemma 3. For $0<s \leq 1 / 2$ and $m=\lfloor n / 2\rfloor$, we have

$$
r(m, s, 0)=\max \{r(i, s, 0): 1 \leq i \leq m\} .
$$

Proof. By (11), we have

$$
\frac{\partial}{\partial i}\left(2 r(i, s, 0)^{2}\right)=\frac{N_{1} N_{2}}{D},
$$

where

$$
\begin{aligned}
N_{1} & =\left(s^{2}-s+1\right)^{2}, \\
N_{2} & =(1-2 s) i^{2}-2 n(1-s)^{2} i+n^{2}(1-s)^{2}, \\
D & =(1-s)^{2} n+((n-i)-(1-2 s)) i .
\end{aligned}
$$

We will show that $r(i, s, 0)$ is an increasing function of $i$. It is easy to see that $N_{1} / D>0$. So, it suffices to show $N_{2} \geq 0$. Fix $s, n$, and let $f(i):=N_{2}$. If $s=1 / 2$, then $f(i)=n(n-2 i) / 4 \geq 0$, as desired. If $s<1 / 2$, then $f(i)$ is a quadratic function of $i$ with positive coefficient for $i^{2}$. Since $f(i)$ takes minimum at $i=n(1-s)^{2} /(1-2 s)>n / 2$ and $f(n / 2)=n^{2}(1-2 s) / 4>0$, we can conclude that $f(i)>0$ for $1 \leq i \leq n / 2$.

Lemma 4. Let $n$ be even and let $m=n / 2$. Then we have

$$
\min \{r(m, s, 0): s \in[0,1]\}=r(m, 1 / 2,0),
$$


and

$$
r(m, 1 / 2,0)=\frac{3}{8} \sqrt{\frac{2 n}{n+1}}
$$

Proof. By 111 we have

$$
\frac{\partial}{\partial s}\left(r(n / 2, s, 0)^{2}\right)=\frac{-n(1-2 s)\left(s^{2}-s+1\right)\left(2 s^{2}-2 s+n\right)}{\left(4 s^{2}-4 s+n+2\right)^{2}},
$$

which implies (15). By (11) we have (16).

Lemma 5. Let $n$ be odd and let $m=\lfloor n / 2\rfloor$. Let $s_{0} \in(0,1)$ be a unique real root of a the cubic equation

$$
4 n X^{3}-6(n+1) X^{2}+2\left(n^{2}+n+2\right) X-n^{2}+1=0 .
$$

Then we have

$$
\min \{r(m, s, 0): s \in[0,1]\}=r\left(m, s_{0}, 0\right),
$$

and

$$
\max \left\{r(m, s, s): s \in\left[s_{0}, 1 / 2\right]\right\}=r\left(m, s_{0}, s_{0}\right) .
$$

Moreover, $r\left(m, s_{0}, 0\right)^{2}$ is a unique real root of the following cubic equation with integral coefficients:

$$
2048(n+1) n^{3} X^{3}+a_{2} X^{2}+a_{1} X+a_{0}=0,
$$

where

$$
\begin{aligned}
& a_{0}=-9\left(n^{2}-1\right)^{2}\left(n^{4}-4 n^{3}+2 n^{2}+4 n+13\right), \\
& a_{1}=16\left(n^{2}-1\right)\left(2 n^{6}-6 n^{5}-15 n^{4}+38 n^{3}+42 n^{2}+48 n-29\right), \\
& a_{2}=64\left(8 n^{6}-8 n^{5}-41 n^{4}-28 n^{3}-10 n^{2}+36 n+27\right) .
\end{aligned}
$$

Proof. We start with (11). Then we get (18) by computing $\frac{\partial}{\partial s}\left(r(m, s, 0)^{2}\right)$. Moreover, it follows from (17) that $1 / 2-1 / n^{2}<s_{0}<1 / 2$ for $n \geq 3$. In fact, we have

$$
s_{0}=\frac{1}{2}-\frac{3}{4 n^{2}}-\frac{3}{8 n^{3}}+O\left(n^{-4}\right) .
$$

By (10) we have $2 r(m, s, s)^{2}=\left(2 s^{2}-2 s+1\right) m /(1+m)$, which implies (19).

Finally we outline how to show that $r\left(m, s_{0}, 0\right)^{2}$ satisfies (20). This can be done by direct computation (with aid of computer). By (11) we have

$$
2 r(m, s, 0)^{2}=\frac{m(1+m)\left(s^{2}-s+1\right)^{2}}{m^{2}+(1-s)^{2}+2 m\left(s^{2}-s+1\right)} .
$$

The numerator of $r\left(m, s_{0}, 0\right)^{2}$ is a biquadratic polynomial of $s_{0}$, and one can reduce it to a quadratic polynomial using (17). Substitute the reduced $r\left(m, s_{0}, 0\right)^{2}$ into 200 , and reduce these fractions to a common denominator. Then one can check that the numerator vanishes by reducing it using (17). 
Define

$$
s^{*}= \begin{cases}1 / 2 & \text { if } n \text { is even, } \\ s_{0} & \text { if } n \text { is odd }\end{cases}
$$

Lemma 6. Let $m=\lfloor n / 2\rfloor$, and let $V\left(S_{n}\right)=\{p\} \cup X \cup Y$ be a proper partition with $i=|X| \leq m$. Then $S_{n} \cap H_{p}\left(X, Y, s^{*}, t\right)$ is contained in a hyperdisk of radius $r\left(m, s^{*}, 0\right)$ for all $t \in\left[s^{*}-1, s^{*}\right]$.

Proof. We have $r\left(i, s^{*}, t\right) \leq r\left(i, s^{*}, 0\right) \leq r\left(m, s^{*}, 0\right)$, where the first inequality follows from Lemma 2 , and the second one from Lemma3.

Theorem 4. Let $m=\lfloor n / 2\rfloor$, and define $s^{*}$ by (23). Then we have $\gamma\left(n, B_{n-1}\right)=$ $r\left(m, s^{*}, 0\right)$.

For $n=2 m$, it follows from (16) that

$$
r(m, 1 / 2,0)=\frac{3}{4 \sqrt{2}}\left(1-\frac{1}{2 n}+\frac{3}{8 n^{2}}-\frac{5}{16 n^{3}}+O\left(n^{-4}\right)\right) .
$$

On the other hand, for $n=2 m+1$, it follows from (20) that

$$
r\left(m, s_{0}, 0\right)=\frac{3}{4 \sqrt{2}}\left(1-\frac{1}{2 n}+\frac{3}{8 n^{2}}-\frac{13}{16 n^{3}}+O\left(n^{-4}\right)\right) .
$$

One can also get (25) by substituting (21) directly into (22). By Theorem 4 with (16), (24) and (25), we have the following.

\section{Corollary 5.}

$$
\gamma\left(n, B_{n-1}\right)= \begin{cases}\frac{3}{4} \sqrt{\frac{2 n}{n+1}} & \text { if } n \text { is even, } \\ \frac{3}{4} \sqrt{\frac{2 n}{n+1}}-\frac{3 \sqrt{2}}{8 n^{3}}+O\left(n^{-4}\right) & \text { if } n \text { is odd }\end{cases}
$$

and

$$
\lim _{n \rightarrow \infty} \gamma\left(n, B_{n-1}\right)=\frac{3 \sqrt{2}}{4} .
$$

Proof of Theorem 4 Let $D$ be a hyperdisk on a hyperplane in $\mathbb{R}^{n}$ through which $S_{n}$ can pass. We may suppose that the vertices $p_{1}, p_{2}, \ldots, p_{n+1}$ can pass through $D$ one by one in this order. (If this seems to be impossible, then by replacing $D$ with a hyperdisk of radius $r(D)+\varepsilon$, this would become possible, where $\varepsilon>0$ can be chosen arbitrarily small.) Consider the moment when $p_{m+1}$ passes through $D$. By Lemma 1, $r(D)+\varepsilon$ is at least $r(m, s, 0)$ for some $0<s<1$, which is at least $r\left(m, s^{*}, 0\right)$ by (15) and (18). Thus $r(D)+\varepsilon \geq r\left(m, s^{*}, 0\right)$. Since $\varepsilon$ can be chosen arbitrarily small, we have $r(D) \geq r\left(m, s^{*}, 0\right)$, and hence $\gamma\left(n, B_{n-1}\right) \geq 2 r\left(m, s^{*}, 0\right)$.

Next we show $\gamma\left(n, B_{n-1}\right) \leq 2 r\left(m, s^{*}, 0\right)$. Instead of pushing $S_{n}$ through the hole, we fix our $S_{n}$ and move the hyperplane containing the hole. Let $D$ be the hyperdisk of radius $r\left(m, s^{*}, 0\right)$ in the moving wall hyperplane. Let 
$V\left(S_{n}\right)=\left\{p_{1}, \ldots, p_{n+1}\right\}, X_{i}=\left\{p_{1}, \ldots, p_{i}\right\}$, and $Y_{i}=\left\{p_{i+2}, \ldots, p_{n+1}\right\}$. Since $\left\{p_{i+2}\right\} \cup X_{i+1} \cup Y_{i+1}$ is also a proper partition, we have $H_{p_{i+1}}\left(X_{i}, Y_{i}, s^{*}, s^{*}\right)=$ $H_{p_{i+2}}\left(X_{i+1}, Y_{i+1}, s^{*}, s^{*}-1\right)$.

Let $H_{i}=H\left(X_{i}, Y_{i}, s^{*}, s^{*}-1\right)$. Suppose that our hole $D$ sits in $H_{i}$ for some $1 \leq i \leq m$. By rotating this wall hyperplane around $I\left(X_{i}, Y_{i}, s^{*}\right)$, we get $H\left(X_{i}, Y_{i}, s^{*}, s^{*}\right)=H_{i+1}$. By Lemma 6, we may assume that, in this rotation process, the intersection of the fixed $S_{n}$ and the wall hyperplane is always contained in $D$. Namely, we can move the wall hyperplane from $H_{i}$ to $H_{i+1}$ so that the intersection of $S_{n}$ and the wall is always contained in the hole.

Therefore, starting from $H_{1}$, we can send the wall hyperplane to the position of $H_{m+1}$. If $n$ is even, then in the process from $H_{m}$ to $H_{m+1}$, we get $H\left(X_{m}, Y_{m}, 1 / 2,0\right)$. This hyperplane divides $S_{n}$ into two parts that are mutually congruent, and we are done (by repeating the same procedure in the reverse order). If $n$ is odd, then by translating $H_{m+1}=H\left(X_{m}, Y_{m}, s^{*}, s^{*}\right)$, we get $H\left(X_{m}, Y_{m}, 1 / 2,1 / 2\right)$, which divides $S_{n}$ into mutually congruent parts. Moreover, by (19), the intersection of $S_{n}$ and the hyperplane is always contained in $D$ in the process of this translation. This completes the proof.

3.3. Holes of shape $Q_{n-1}$. In [11] the following is proved: for every $\varepsilon>0$ there is an $N$ such that for every $n>N$ one has

$$
S_{n} \subset(2+\varepsilon) Q_{n} \text {. }
$$

This gives

$$
\lim _{n \rightarrow \infty} \Gamma\left(n, Q_{n-1}\right) \leq 2 .
$$

Since $Q_{n-1} \subset B_{n-1}$, we get lower bounds for $\gamma\left(n, Q_{n-1}\right)$ and $\Gamma\left(n, Q_{n-1}\right)$ from $\gamma\left(n, B_{n-1}\right)$ and $\Gamma\left(n, B_{n-1}\right)$, respectively. Here we include a simple proof of the following slightly weaker bound for $\Gamma\left(n, Q_{n-1}\right)$.

Theorem 6. We have

$$
\Gamma\left(n, Q_{n-1}\right) \geq \sqrt{\frac{2(n-1)}{n+1}},
$$

with equality holding iff there exists an Hadamard matrix of order $n+1$.

Proof. Let $d=\Gamma\left(n, Q_{n-1}\right)$. Then $S_{n}$ can pass through a hole of $d Q_{n-1}$ by translation. So (2) and (1) imply

$$
\operatorname{width}\left(d Q_{n-1}\right)=\frac{d}{\sqrt{n-1}} \geq \operatorname{width}\left(S_{n}\right) \geq \sqrt{\frac{2}{n+1}},
$$

which gives (26). Moreover, if $S_{n} \subset \ell Q_{n}$, then we have

$$
\ell \geq \frac{\sqrt{n}}{\sqrt{n-1}} \Gamma\left(n, Q_{n-1}\right) \geq \sqrt{\frac{2 n}{n+1}} .
$$


It is known that $\ell=\sqrt{(2 n) /(n+1)}$ iff there exists an Hadamard matrix of order $n+1$, see e.g., [13].

Problem 2. Do we have $\gamma\left(n, Q_{n-1}\right)=\Gamma\left(n, Q_{n-1}\right)=\sqrt{2}-o(1)$ ?

3.4. Holes having minimum volumes. In [7], the following problem is posed.

Problem 3. Find the minimum $(n-1)$-dimensional volume of a compact hole in a hyperplane of $\mathbb{R}^{n}$ such that $S_{n}$ can pass through it.

The following variation seems to be easier.

Problem 4. Find the minimum $(n-1)$-dimensional volume of a compact hole in a hyperplane of $\mathbb{R}^{n}$ such that $S_{n}$ can pass through it by translation perpendicular to the hyperplane.

We list possible candidates. Put $\sqrt{2} S_{n}$ in $\mathbb{R}^{n+1}$ so that the vertices are $e_{1}, \ldots, e_{n+1}$, where $e_{i}$ is the $i$-th standard base of $\mathbb{R}^{n+1}$.

Project the $\sqrt{2} S_{n}$ in the direction of

$$
(1,-1, \overbrace{0, \ldots, 0}^{n-1})
$$

Then the hole created by the shadow has volume

$$
\frac{1}{(n-1) !} \sqrt{\frac{n+1}{2}} .
$$

Next suppose that $n$ is odd and write $n=2 k+1$. Project the $\sqrt{2} S_{n}$ in the direction of

$$
(\overbrace{1, \ldots, 1}^{k+1}, \overbrace{-1, \ldots,-1}^{k+1})
$$

Then the corresponding hole has volume

$$
\frac{2}{(n-1) !} \text {. }
$$

Finally suppose that $n$ is even and write $n=2 k$. Project the $\sqrt{2} S_{n}$ in the direction of

$$
(\overbrace{k+1, \ldots, k+1}^{k}, \overbrace{-k, \ldots,-k}^{k+1})
$$

In this case, the volume of the hole is

$$
\frac{2}{(n-1) !} \sqrt{\frac{n}{n+2}} \text {. }
$$

Among the above examples, the smallest one is 27 for $n \leq 5$. For $n=7$, (27) and (28) coincide. For the other cases, (28) and (29) give the smallest one. 


\section{ACKNOWLEDGMENT}

We thank Keith Ball, Matthieu Fradelizi, and Jiří Matoušek for their valuable comments.

\section{REFERENCES}

[1] I. Bárány, H. Maehara, N. Tokushige. Tetrahedra passing through a triangular hole. submitted.

[2] R. Brandenberg, T. Theobald. Radii of simplices and some applications to geometric inequalities. Beiträge Algebra Geom. 45 (2004) 581-594.

[3] R. Brandenberg, T. Theobald. Radii minimal projections of polytopes and constrained optimization of symmetric polynomials. Adv. Geom. 6 (2006) 71-83.

[4] H. T. Croft, K. J. Falconer, R. K. Guy. Unsolved Problems in Geometry, SpringerVerlag, New York, 1991.

[5] H. E. Debrunner, P. Mani-Levitska. Can you cover your shadows? Discrete Comput. Geom. 1 (1986) 45-58.

[6] M. Fradelizi. Hyperplane sections of convex bodies in isotropic position. Beiträge Algebra Geom. 40 (1999) 163-183.

[7] J. Itoh, T. Zamfirescu. Simplices passing through a hole, J. Geom. 83 (2005) 65-70.

[8] J. Itoh, Y. Tanoue, T. Zamfirescu. Tetrahedra passing through a circular or square hole, Rend. Circ. Mat. Palermo (2) Suppl. No. 77 (2006) 349-354.

[9] G. Kós, J. Törőcsik. Convex disks can cover their shadow. Discrete Comput. Geom. 5 (1990) 529-531.

[10] M.D. Kovalyov, Covering a convex figure by its images under dilataion, Ukrainskiy Mat. Sbornik, 27/84 (1984), 57-68. (in Russian)

[11] H. Maehara, I. Ruzsa, N. Tokushige. Large regular simplices contained in a hypercube. Period. Math. Hungarica, 58 (2009) 121-126.

[12] S. V. Pukhov. Kolmogorov diameters of a regular simplex. Mosc. Univ. Math. Bull. 35 (1980) 38-41.

[13] I. J. Schoenberg. Regular simplices and quadratic forms. Journal of the London Mathematical Society 12 (1937) 48-55.

[14] P. Steinhagen. Über die grösste Kugel in einer konvexen Punktmenge. Abh. Math. Sem. Hamburg, 1 (1921) 15-26.

[15] B. Weißbach. Über die senkrechten Projektionen regulär Simplexe. Beitr. Algebra Geom. 15 (1983) 35-41.

[16] T. Zamfirescu. How to hold a convex body, Geometrae Dedicata 54 (1995) 313-316.

[17] K. Zindler. Über konvexe Gebilde, Monatsh. Math. Physik 30 (1920) 87-102.

Research Institute of Educational DeVelopment, Tokai University, 228-4 TOMIGAYA, SHIBUYA-KU, TOKYO, 151-8677, JAPAN

E-mail address: maehara@tokai-u.jp

College of Education, Ryukyu University, Nishihara, OKinawa, 9030213 JAPAN

E-mail address: hide@edu.u-ryukyu.ac.jp 